\title{
MR imaging in pulmonary embolism: does it have a future?
}

\author{
Francesco Sardanelli $\cdot$ H. Dirk Sostman
}

Received: 17 August 2014/Accepted: 2 September 2014/Published online: 16 September 2014

(C) Italian Association of Nuclear Medicine and Molecular Imaging 2014

In most centers, computed tomography (CT) pulmonary angiography (CTPA) is the first-choice imaging test for diagnosing acute pulmonary embolism (PE). However, CT may be undesirable, contraindicated or inconclusive. In such cases, the usual alternatives include scintigraphy and magnetic resonance (MR) imaging. Scintigraphy is better validated and is discussed extensively in this issue therefore some consideration of MR is warranted.

Despite the acknowledged imperfections of scintigraphy, MR imaging has failed to compete with it in most centers. Past publications on MR imaging in PE have revealed variable (but usually mediocre) diagnostic accuracy, and a high rate of non-diagnostic examinations due to motion artifacts or poor enhancement. In addition, MR is not always easily available and it can be very difficult to perform an MR study when the patient is acutely ill and dyspneic. Research in MR imaging continues, however, and recent data should make it possible to assess whether technical progress with MR has made the technique more competitive with respect to scintigraphy or even CTPA.

A 2013 publication evaluated four MR techniques in 277 patients with a CTPA reference standard [1]. The MR techniques included: unenhanced free-breathing as well as breath-hold-ECG-gated techniques and contrast-enhanced perfusion and MR angiography (MRA). The sensitivity of

\section{F. Sardanelli ( $\square)$}

Department of Biomedical Sciences for Health, IRCCS

Policlinico San Donato, Unit of Radiology, Università degli

Studi di Milano, Via Morandi 30, San Donato Milanese,

20097 Milan, Italy

e-mail: francesco.sardanelli@unimi.it

H. D. Sostman

Department of Radiology, Weill Cornell Medical College, 1300

York Ave, New York, NY, USA the best MR sequence (corresponding to the better reading between those of two expert radiologists) did not exceed $90 \%$. With the observed disease prevalence of $37.5 \%$, the negative predictive values for this sequence ranged from 87 to $91 \%$ and for other sequences were as low as $80 \%$. However, up to $50 \%$ of contrast-enhanced MRA studies were technically inadequate (30\% due to artifacts, $20 \%$ due to poor enhancement). If we consider inconclusive studies as negative tests, the sensitivity drops to $44 \%$ and the negative predictive value to $74 \%$. If we exclude inconclusive studies, the diagnostic yield is unacceptably low. On the other hand, if a test with this sensitivity was applied to a population with a $10 \%$ disease prevalence (which is common in many USA emergency departments), $30 \%$ or more of the positive tests would be false positive. Importantly, the MR perfusion sequences had negative predictive values of less than $85 \%$, therefore a negative MR perfusion examination could not rule out PE in the same way that negative pulmonary scintigraphy has been proven to do.

These results are comparable to some of the less good data obtained with pulmonary scintigraphy. Why then should one consider MR as an alternative to $\mathrm{CT}$, rather than simply using nuclear medicine? We think the most persuasive reason is the flexibility offered by multiple MR techniques.

When PE is highly probable clinically $(70-80 \%$ pretest probability), a negative CTPA is insufficient to exclude PE (the post-test probability remains about $30 \%$ ). At this point MR can be used as a problem-solving test, in addition to examine the pulmonary arteries; indeed, contrastenhanced MR can obtain indirect venography to evaluate for deep venous thrombosis. If this, too, is negative, then $\mathrm{PE}$ is less likely, but if it is positive, venous thromboembolism is documented. Indirect venography can also be 
performed following CTPA, but in today's typical production-line imaging environment, the patient has often been discharged before the CTPA is reviewed.

We can also consider MR as an alternative test when CTPA is undesirable (stage 3 or greater renal failure, severe allergy to iodinated contrast material, pregnancy). However, in these conditions we should try to exploit the potential of non-contrast MR approaches before injecting gadolinium. There has been progressive refinement of both bright-blood and black-blood sequences, mainly in the form of balanced steady-state-free precession (b-SSFP) and half-Fourier acquisition single-shot turbo spin-echo (HASTE) sequences, respectively. Parallel imaging has represented a further improvement, allowing higher spatial and/or temporal resolution. The good anatomical rendering and the optimal contrast between laminar blood flow and clot of b-SSFP images make this approach very promising for PE diagnosis. Importantly, Revel et al. [1] showed that, when excluding PE limited to the segmental or sub-segmental levels, b-SSFP sequences, either free-breathing or ECG-gated breath-hold, allowed $100 \%$ sensitivity for PE by both readers. The authors pointed out that these MR sequences can be repeated rapidly until optimal image quality is obtained. This approach should be tested in further clinical studies.

The flexibility inherent in MR is exemplified by the recent work of Hosch et al. [2], who used a stepwise MR protocol in 45 patients. The MR techniques used included: (1) bright-blood free-breathing non-ECG-gated b-SSFP sequence; (2) black-blood breath-hold and ECG-gated HASTE sequence; (3) first pass perfusion breath-hold gradient-echo volume acquisition during injection of contrast material; (4) breath-hold high spatial resolution MRA with a second bolus of contrast material; and (5) volumeinterpolated breath-hold examination (VIBE) sequence. The authors were able to obtain the complete MR protocol in $40 / 45$ patients $(88 \%)$. Of note, however, in the remaining five patients with severe dyspnea PE was diagnosed using only the b-SSFP sequences. For central and lobar PE, sensitivity ranged from $76 \%$ for VIBE to $98 \%$ for perfusion; specificity ranged from 89 (perfusion and HASTE) to $100 \%$ (angiography). For segmental PE, sensitivity ranged from $46 \%$ for HASTE to $95 \%$ for perfusion; specificity ranged from 82 (perfusion) to $97 \%$ (angiography). While the value demonstrated for MR perfusion images again raises the question "why not just use perfusion scintigraphy?" the additional specificity provided by angiography answers the question in favor of MR. Although this study has some limitations (small sample size, unclear independent reading of reference standard, requirement for double bolus of contrast material), it shows the potential diagnostic power of MR. A simple MR protocol including only three-plane b-SSFP and coronal perfusion sequences (a less than 10 min examination) could be stopped at the first step in the event of immediate diagnosis of central/lobular PE with b-SSFP, but could be continued to include angiography or even indirect venography to exclude PE.

For acute PE, MR remains an alternative to CTPA only for selected cases. In addition to the uncertainty about the best diagnostic MR protocol, the performance of an adequate MR study in the acute clinical setting requires stateof-the-art equipment as well as staff (both radiologists and technologists) who are expert in thoracic/cardiac MR. These conditions are rare enough at the best of times, but even less likely to be met in emergency or on-call services. In such circumstances the technical robustness, long clinical experience and (often but not always) greater availability of nuclear medicine techniques render scintigraphy usually a better option than MR in clinical practice today.

For chronic PE, in the absence of acute illness, the scenario is quite different. MR can be performed in optimal conditions and is competitive both with CTPA and with perfusion scintigraphy. A recent study [3] evaluated 132 patients, 78 of whom (59\%) had a final diagnosis of chronic thromboembolic pulmonary hypertension. Sensitivity and specificity were 97 and $92 \%$ for MR perfusion, 94 and $98 \%$ for CTPA, and 96 and $90 \%$ for perfusion scintigraphy (no cases of surgically accessible disease were missed with any modality). Here the advantage of MR is that it is a radiation-free imaging modality that can delineate both perfusion and vascular anatomy. If these results are confirmed, MR may become a first-choice examination for chronic PE, especially if one considers the possibility of performing functional cardiac MR in the same diagnostic session and the probable need for repeated examinations as the patient is followed through therapy.

We should remember that CT technology continues to evolve rapidly with the advent of hundreds of detector rows, dual source, dual energy, iterative reconstruction algorithms and many other developments. Together with other advances, this evolution is leading to a progressive reduction of ionizing radiation exposure. As the diagnostic accuracy, technical robustness and widespread availability of CT is combined with continuously enhanced safety, we doubt that MR will ever challenge it as a first-line modality for PE imaging.

So what about the choice between MR and scintigraphy as a problem-solving modality? On the basis of our own experience and the published data of others, we feel confident in asserting that imaging diagnosis of PE performed with MR or scintigraphy can give either superb or execrable results. We cannot emphasize strong enough that the choice of modality should always be made on the basis of local 
expertise. Both modalities have future possibilities. In the future development of scintigraphy, refinements of SPECT and fusion techniques are promising, the exploration of PET is paradoxically intriguing, and the development of highperformance thrombus-avid radiopharmaceuticals would be a transformational event if it can be achieved. For MR, some of the approaches mentioned above are now worthy of larger clinical trials.

There is a future for MR in diagnosing PE, but the competition it faces will be increasingly stiff. And that, in itself, is a wonderful thing for our patients.

Conflict of interest Francesco Sardanelli and H. Dirk Sostman declare no conflicts of interest.

\section{References}

1. Revel MP, Sanchez O, Lefort C, Meyer G, Couchon S, Hernigou A, Niarra R, Chatellier G, Frija G (2013) Diagnostic accuracy of unenhanced, contrast-enhanced perfusion and angiographic MRI sequences for pulmonary embolism diagnosis: results of independent sequence readings. Eur Radiol 23:2374-2382

2. Hosch W, Schlieter M, Ley S, Heye T, Kauczor HU, Libicher M (2014) Detection of acute pulmonary embolism: feasibility of diagnostic accuracy of MRI using a stepwise protocol. Emerg Radiol 21:151-158

3. Rajaram S, Swift AJ, Telfer A, Hurdman J, Marshall H, Lorenz E, Capener D, Davies C, Hill C, Elliot C, Condliffe R, Wild JM, Kiely DG (2013) 3D contrast-enhanced lung perfusion MRI is an effective screening tool for chronic thromboembolic pulmonary hypertension: results from the ASPIRE Registry. Thorax 68:677-678 